\title{
Work-related physical and psychosocial risk factors for sick leave in patients with neck or upper extremity complaints
}

\author{
Sandra D. M. Bot $\cdot$ Caroline B. Terwee $\cdot$ \\ Daniëlle A. W. M. van der Windt • \\ Allard J. van der Beek · Lex M. Bouter · Joost Dekker
}

Received: 6 February 2006 / Accepted: 1 March 2007 / Published online: 5 April 2007

(c) Springer-Verlag 2007

\begin{abstract}
Objectives To study work-related physical and psychosocial risk factors for sick leave among patients who have visited their general practitioner for neck or upper extremity complaints.

Methods Three hundred and forty two patients with neck or upper extremity complaints completed self-report questionnaires at baseline and after 3 months. Cox regression models were used to investigate the association between work-related risk factors and sick leave (i.e., lost days from work due to neck or upper extremity complaints in 3 months). Effect modification by sick leave at baseline,
\end{abstract}

S. D. M. Bot $(\bowtie) \cdot$ C. B. Terwee

Daniëlle A. W. M. van der Windt · A. J. van der Beek .

L. M. Bouter · J. Dekker

Institute for Research in Extramural Medicine (EMGO Institute),

VU University Medical Center, Van der Boechorststraat 7,

1081 BT Amsterdam, The Netherlands

e-mail: s.bot@vumc.nl

Daniëlle A. W. M. van der Windt

Department of General Practice,

VU University Medical Center,

Amsterdam, The Netherlands

A. J. van der Beek

Department of Public and Occupational Health,

VU University Medical Center,

Amsterdam, The Netherlands

A. J. van der Beek

Body@Work, Research Center Physical Activity,

Work And Health, TNO,

Amsterdam, The Netherlands

J. Dekker

Department of Rehabilitation Medicine,

VU University Medical Center,

Amsterdam, The Netherlands sex, worrying and musculoskeletal co-morbidity was evaluated by adding product terms to the regression models.

Results In the subgroup of patients who scored high on the pain copying scale "worrying" the hazard ratio of sick leave was 1.32 (95\% CI 1.07-1.62) per 10\% increase in heavy physical work. The subgroup of patients who were sitting for long periods of time had a reduced risk of sick leave as compared to patients who did not spend a lot of time sitting, again only in patients who scored high on the pain coping scale "worrying" (adjusted HR $=0.17,95 \%$-CI 0.04-0.72). Other work-related risk factors were not significantly related to sick leave.

Conclusions Heavy physical work increased the risk of sick leave and prolonged sitting reduced the risk of sick leave in a subgroup of patients who worried much about their pain. Additional large longitudinal studies of sufficiently large size among employees with neck or upper extremity complaints are needed to confirm our results.

Keywords Cohort study · Sick leave · Workload · Neck · Upper extremity

\section{Introduction}

Many people suffer from work-related neck and upper extremity complaints. In a population-based study in the Netherlands, the 12-month prevalence of work-related neck and upper extremity symptoms was found to be $31 \%$ (Blatter and Bongers 1999). These complaints have an important impact on loss of time from work. Sick leave has enormous cost implications, considering costs to employers, insurers, health care providers, and patients themselves. In the Netherlands, $8 \%$ of the employed population reported lost days from work in the past year because of complaints at the 
neck, shoulder, arm, elbow, wrist or hand (Blatter et al. 2001). The results of a British national survey in 1995 showed that musculoskeletal disorders of the neck and upper extremity were responsible for the loss of 4.2 million working days in a 12-month period (Jones et al. 1998). Borghouts et al. (1999) estimated the total number of sick days due to neck pain in 1996 in the Netherlands to be 1.4 million, with a total cost of US\$ 185.4 million.

It is well recognized that neck and upper extremity complaints have a multi-factorial aetiology. Several reviews have identified individual characteristics, and (work-related) physical and psychosocial factors as risk factors for the onset of neck and upper extremity complaints (Bernard 1997; Bongers et al. 2002a, b; Malchaire et al. 2001; Muggleton et al. 1999; van der Windt et al. 2000; Zakaria et al 2002). Furthermore, these factors have also been recognized as potential prognostic factors for chronic pain and disability in patients with neck or upper extremity complaints (Ariëns et al. 2000, 2001; Cole and Hudak 1996; Eriksen et al. 1999; Feuerstein et al. 2000). However, relatively little is known about the association of work-related factors with sick leave in patients with neck or upper extremity complaints. Risk factors for the onset of neck and upper extremity complaints may differ from risk factors for sick leave due to these complaints (IJzelenberg et al. 2004). Ekberg and Wildhagen (1996) studied the impact of physical workload, work organization, psychosocial conditions and individual characteristics on days of sick leave after rehabilitation in patients with neck and shoulder disorders. They found two work-related factors (i.e., work content and uncomfortable sitting) to be independently related to sick leave days. A prospective cohort study on work-related determinants of sick leave in employees without neck pain at baseline suggested that work-related neck flexion and rotation, sitting, decision authority and skill discretion were independently related to sick leave due to neck pain (Ariëns et al. 2002). In contrast to these findings, a recent cross-sectional study on risk factors for musculoskeletal sick leave showed that physical workload nor psychosocial workload were significantly related to sick leave due to neck or upper extremity pain (IJzelenberg et al. 2004). Hansson and Jensen (2004) reviewed the literature on the causes for back and neck disorders and found that heavy physical workload, bent or twisted working position and low work satisfaction increased the risk of short-term and long-term sick leave, and self-reported pain and functional impairments were associated with a high risk for long-term sick leave.

Exposure to work-related physical risk factors may lead to pain and disability, which in turn may lead to sick leave. Psychosocial exposures at work may lead to psychological distress that may cause physiologic changes in the neck and upper extremity (e.g., increased muscle tension (Hagg and
Astrom 1997; Lundberg 2002; Rissen et al. 2000), causing pain and disability, which in turn may lead to sick leave. Furthermore, psychosocial exposures at work may modify the effect of physical workload on sick leave. The objective of our study was to determine the influence of work-related physical and psychosocial risk factors on sick leave due to neck or upper extremity complaints in patients who have visited their general practitioner (GP) for these complaints.

\section{Methods}

Design and study population

This study is part of a large observational cohort study on musculoskeletal disorders conducted in 61 general practices (97 GPs) (van der Waal et al. 2003). Part of the GPs participated in the second Dutch National Survey of General Practice (NS2), carried out by the Netherlands Institute for Health Services Research in co-operation with the National Information Network of General Practice in 2001 (Schellevis et al. 2003). The Medical Ethics Committee of the VU University Medical Center approved our study. Written informed consent was obtained from all patients. Details of the study design are described elsewhere (van der Waal et al 2003).

Over a period of 1 year the GPs recruited 727 patients with a new complaint or new episode of a complaint at the neck, shoulder, elbow, arm, wrist or hand. An episode was considered to be 'new' if patients had not visited their GP for the same complaint during the preceding 3 months. Patients were eligible for participation if they were 18 years or older and capable of filling in Dutch questionnaires. Patients were excluded from the study if the presented symptoms were presumably caused by a fracture, malignancy, prosthesis, amputation or congenital defect or if the patient was pregnant.

At baseline a self-report questionnaire was sent to collect data on a broad range of factors (including work-related factors) that may be predictive of sick leave. In total, 643 patients $(88 \%)$ returned the baseline questionnaire, of whom five patients were excluded (two were too young; three were pregnant). For our study, patients who reported to have paid work for more than $8 \mathrm{~h}$ per week were included (8-16 h: $n=41 ; \quad 17-24 \mathrm{~h}: \quad n=69 ; \quad 25-36 \mathrm{~h}$ : $n=101 ;>36$ h: $n=172$ ). Outcome (sick leave due to neck and/or upper extremity complaints) was assessed after 3 months using a self-report questionnaire.

\section{Outcome measure}

Sick leave was measured at 3 months after baseline by the question: "how long were you absent from work because of 
your complaint since the baseline questionnaire". Response categories were (1) no sick leave; (2) less than 1 week; (3) between 1 and 2 weeks; (4) between 2 weeks and 1 month; (5) between 1 and 3 months; (6) more than 3 months. Sick leave was dichotomised into no sick leave (response category 1) and sick leave (response categories 2-6).

\section{Determinants}

At baseline, work-related physical risk factors were assessed by a questionnaire consisting of two scales "heavy physical workload" [12 items; e.g., does your work involve (a) standing for long periods of time, (b) walking for long periods of time, (c) moving loads, (d) physical hard work] and "long-lasting postures and repetitive movements" [six items; e.g., does your work involve (a) making the same movement for long periods of time; (b) bending or twisting your neck often; (c) working in the same position for long periods of time; (d) doing repetitive tasks with arms, hands or fingers many times per minute) (Bot et al. 2004). Response options varied from "seldom or never", "sometimes", "often", and "(almost) always". Scoring was done by simply adding up the response to each item, which produced a raw score from 0 to 36 for the first subscale, and 0 to 18 for the second subscale. The final scores were calculated by dividing the raw score by the maximum score, multiplied by 100 , resulting in a final score ranging between 0 (no workload) and 100 (highest workload) for both subscales. The internal consistency and validity of this questionnaire were considered to be good in a population with upper extremity and lower extremity musculoskeletal disorders (Bot et al. 2004). Furthermore, two questions about "prolonged sitting" and " working with visual display units (VDU) for long periods of time" (yes/no) were asked.

Work-related psychosocial risk factors were assessed according to the Demands-Control model of Karasek (1979). We used three subscales of the Job Content Questionnaire (JCQ): "job demands", "decision authority" and "co-worker support" and one single item on "job security" (Karasek et al. 1998). The hypothesis of the DemandsControl model is that the most adverse reactions of psychological strain occur when demands are high and the worker's decision latitude is low (Karasek et al. 1998). Therefore, we decided to combine the subscales high job demands and low decision authority into an additional determinant "job strain". Both subscales were dichotomized by their median score and four categories were created: (1) high decision authority and low job demands (reference category); (2) high decision authority and high job demands; (3) low decision authority and low job demands; (4) low decision authority and high job demands.
Statistical analysis

Descriptive statistics (e.g., means and standard deviations) were used to present baseline characteristics of the study population. Differences between patients reporting sick leave and patients without sick leave were tested with the unpaired Student $T$-test (continuous variables) and the Chi square $\left(\chi^{2}\right)$ test (categorical and dichotomous variables). In case a continuous variable was not normally distributed the Mann-Whitney test was used.

Instead of using logistic regression analysis, Cox regression models with equal survival time were used to investigate the association between work-related risk factors (determinants) and sick leave (outcome). Hazard ratios are more easy to interpret and provide a better estimate of relative risks than odds ratios (Barros and Hirakata 2003; Lee and Chia 1994; Skov et al. 1998; Thompson et al. 1998) Associations were expressed as hazard ratios (HR) and corresponding $95 \%$ confidence intervals $(95 \% \mathrm{CI})$ per unit increase of the risk factor involved. For continuous factors the linearity of the relation between the risk factor and sick leave was examined. Factors that were found to be non-linearly related to sick leave were either dichotomized or divided into tertiles with equally sized groups (Table 1). The "low-category" served as the reference category in all analyses.

To prevent multicollinearity we checked whether the psychosocial risk factors, the physical risk factors, and potential confounders were highly correlated $(P>0.5$ or $P<-0.5)$. The factor with the highest association with sick leave was retained and the other factor was removed from further analyses. This was the case for prolonged sitting and prolonged VDU work $(P=0.69)$, distress and worrying $(P=0.52)$; prolonged VDU-work and heavy physical work $(\mathrm{P}=-0.56)$, and prolonged sitting and heavy physical work $(P=-0.65)$.

We performed the analyses in several stages. First, the unadjusted relationships between the work-related risk factors and sick leave were examined. Second, these relationships were adjusted for each of the potential confounders individually. Potential confounders of the relationship between work-related risk factors and sick leave included individual factors, general health, characteristics of the complaint, and other work-related risk factors (Table 1). Factors were considered as potential confounders if they had been reported as predictive of sick leave or poor prognosis in previously published scientific papers. Only those factors that led to a considerable change in the regression coefficient of the risk factor $(>10 \%)$ were considered as potential confounders in the multiple regression models. Next, a manual forward selection procedure was used to sequentially include potential confounders that induced the most change in the regression coefficient of the risk factor 
Table 1 Potential confounders of the association between work-related risk factors and sick leave in patients with neck or upper extremity complaints

\begin{tabular}{|c|c|}
\hline Potential confounders & Categorization \\
\hline \multicolumn{2}{|l|}{ Individual factors } \\
\hline Age & Continuous (per year) \\
\hline Sex & Male versus female \\
\hline Smoking & Present versus previous smoke \\
\hline Education & $\begin{array}{l}\text { Primary versus secondary; } \\
\text { college/university }\end{array}$ \\
\hline $\begin{array}{l}\text { Pain coping }{ }^{\text {a }} \text { retreating } \\
\text { (seven items) }\end{array}$ & Continuous (scale 7-28) \\
\hline $\begin{array}{l}\text { Pain coping }: \text { worrying } \\
\text { (nine items) }\end{array}$ & Continuous (scale 9-36) \\
\hline Distress (six items) & Continuous (scale 1-9) \\
\hline \multicolumn{2}{|l|}{ General health } \\
\hline ACSM position stand ${ }^{c}$ & Norm not met versus norm met \\
\hline Norm healthy activity & Norm not met versus norm met \\
\hline Perceived health $^{\mathrm{d}}$ & Continuous (scale 1-5) \\
\hline Vitality $^{\mathrm{d}}$ (four items) & Continuous (scale $0-100$ ) \\
\hline Quality of life & Continuous (scale 1-5) \\
\hline \multicolumn{2}{|l|}{ Characteristics of the complaint } \\
\hline Kind of complaint & Localized versus generalized \\
\hline Duration of the complaint & $\begin{array}{l}\text { Categorical }(<1,1-4 \text { weeks; } \\
1-6,>6 \text { months })\end{array}$ \\
\hline $\begin{array}{l}\text { History of neck/upper } \\
\text { limb complaints }\end{array}$ & History versus no history \\
\hline Musculoskeletal co-morbidity & Yes versus no \\
\hline Other co-morbidity & Yes versus no \\
\hline \multicolumn{2}{|l|}{$\begin{array}{l}\text { Work-related psychosocial } \\
\text { factors }(\%)\end{array}$} \\
\hline Decision authority ${ }^{\mathrm{f}}$ (three items) & Tertiles (low, medium, high) \\
\hline Job demands $^{\mathrm{f}}$ (five items) & Tertiles (low, medium, high) \\
\hline Co-worker support ${ }^{\mathrm{f}}$ (four items) & Tertiles (low, medium, high) \\
\hline Job-security ${ }^{\mathrm{f}}$ & $\begin{array}{l}\text { Low security versus } \\
\text { high security }\end{array}$ \\
\hline $\begin{array}{l}\text { Job as perceived } \\
\text { cause of complaint }\end{array}$ & Yes versus no \\
\hline
\end{tabular}

a Pain Coping Inventory (Kraaimaat and Evers 2003; Kraaimaat et al. 1997)

${ }^{\mathrm{b}}$ Four Dimensional Symptom Questionnaire (Terluin 1998)

c American College of Sports Medicine Position Stand (American College of Sports Medicine Position Stand 1990)

${ }^{d}$ Medical Outcomes Study 36-item Short Form Health Survey (Ware and Sherbourne 1992)

e Physical workload questionnaire (Bot et al. 2004)

${ }^{f}$ Job Content Questionnaire (Karasek et al. 1998); \%work-related psychosocial factors were considered as potential confounders in the relation between work-related physical factors and sick leave

(i.e., in order of highest change of the regression coefficient). Confounders that changed the regression coefficient of sick leave by more than $10 \%$ were retained in the model.
If the unadjusted relationship between the work-related risk factor and sick leave was not statistically significant $(P>0.05)$ and remained not significant after controlling for all potential confounders individually, the relationship was adjusted for sick leave at baseline and sex only.

Individual factors may interact in the relation between work-related physical and psychosocial risk factors and sick leave. We considered possible effect modification by sick leave at baseline, sex, coping style worrying (dichotomized) and musculoskeletal co-morbidity. Product terms of the potential effect modifier and the work-related risk factor were added to the model (e.g., sex $\times$ risk factor). Furthermore, possible effect modification by psychosocial risk factors was investigated in the relation between the four physical risk factors and sick leave. Factors significantly interacting with determinants in the model were retained in the models. In case of significant effect modification stratified analyses were carried out presenting the effect of the work-related risk factor for relevant subgroups of workers. All analyses were performed with the use of SPSS for Windows version 10.1 (SPSS Inc., Chicago, IL, USA).

\section{Results}

In total, 342 of the 383 included patients (89\%) completed the 3 months follow-up questionnaire. Fifty-eight of the patients were excluded, because data on sick leave at follow-up were missing. There were no significant differences between responders and dropouts by age, sex, and any of the determinants $(P>0.05)$. Baseline characteristics of patients reporting sick leave in the 3 months following baseline $(n=73)$ and patients without sick leave $(n=252)$ are shown in Table 2 . The mean age of the total study population was 43 years (SD 10.3) and 54\% was female. At baseline 80 patients reported sick leave in the 3 months preceding baseline due to their neck or upper extremity complaint. Half of these patients reported sick leave in the 3 months following baseline. With reference to physical and psychosocial risk factors, there was a statistically significant difference between patients reporting sick leave and patients without sick leave for decision authority, heavy physical work, static postures and repetitive movements, prolonged sitting and prolonged VDU-work.

Table 3 shows the effect of work-related physical and psychosocial risk factors on sick leave. After adjustment for confounders, a statistically significant effect was found for heavy physical work and prolonged sitting, but only in patients with a high score on pain coping strategy "worrying". In patients who scored low on the pain coping subscale "worrying" the hazard ratio of sick leave was 1.05 $(0.93-1.18, P=0.46)$ per $10 \%$ increase in heavy physical 
Table 2 Baseline characteristics and values of patients with neck or upper extremity complaints reporting sick leave $(n=73)$ and of patients reporting no sick leave $(n=252)$ due to their complaints in the 3 months following baseline assessment

$V D U$ visual display unit, $S D$ standard deviation, $I R$ interquartile range

$* P<0.05$; ** $P<0.001$ (independent $T$-test, MannWhitney test, or $\chi^{2}$-test)

a values are percentages of patients unless indicated

\begin{tabular}{|c|c|c|c|}
\hline Characteristic & Sick leave $^{\mathrm{a}}$ & No sick leave ${ }^{a}$ & Total \\
\hline \multicolumn{4}{|l|}{ Individual factors } \\
\hline Age, years [mean (SD)] & $42.0(11.5)$ & $43.6(9.8)$ & $43.0(10.3)$ \\
\hline Sex (female) & $75 \%$ & $49 \% * *$ & $54 \%$ \\
\hline Sick leave in 3 months before baseline & $59 \%$ & $16 \% * *$ & $25 \%$ \\
\hline \multicolumn{4}{|l|}{ Education level } \\
\hline Primary & $48 \%$ & $30 \% *$ & $34 \%$ \\
\hline Secondary & $40 \%$ & $49 \%$ & $48 \%$ \\
\hline College/university & $12 \%$ & $21 \%$ & $18 \%$ \\
\hline \multicolumn{4}{|l|}{ Number of hours work (h) } \\
\hline $8-16$ & $11 \%$ & $12 \%$ & $11 \%$ \\
\hline $17-24$ & $18 \%$ & $25 \%$ & $20 \%$ \\
\hline $25-36$ & $26 \%$ & $30 \%$ & $27 \%$ \\
\hline$>36$ & $45 \%$ & $33 \%$ & $42 \%$ \\
\hline Married/living together & $63 \%$ & $83 \% *$ & $78 \%$ \\
\hline Smoking (now, ever) & $77 \%$ & $67 \%$ & $70 \%$ \\
\hline Pain coping: retreating [scale 7-28; median (IR)] & $11.0(5.0)$ & $9.0(4.8)^{* *}$ & $10.0(4.5)$ \\
\hline Pain coping: worrying (scale 9-36; median (IR)) & $18.0(7.0)$ & $14.0(5.0)^{* *}$ & $15.0(6.0)$ \\
\hline Psychological distress (scale $0-12$; median (IR)) & $7.0(5.0)$ & $4.0(5.0)^{* *}$ & $5.0(6.0)$ \\
\hline \multicolumn{4}{|l|}{ General health } \\
\hline Meeting ACSM position stand & $4 \%$ & $16 \% *$ & $13 \%$ \\
\hline Meeting Norm Healthy Activity & $41 \%$ & $42 \%$ & $43 \%$ \\
\hline Perceived health [scale $1-5$; mean (SD)] & $3.1(0.8)$ & $3.4(0.9)^{*}$ & $3.3(0.8)$ \\
\hline Quality of life [scale 1-5; mean (SD)] & $3.2(0.7)$ & $3.5(0.8)^{*}$ & $3.4(0.8)$ \\
\hline Vitality [scale $0-100 ;$ mean (SD)] & $54.3(20.9)$ & $62.4(17.2)^{* *}$ & $60.0(18.5)$ \\
\hline \multicolumn{4}{|l|}{ Characteristics complaint } \\
\hline Localized complaint & $40 \%$ & $53 \% *$ & $49 \%$ \\
\hline Complaint at both arms & $10 \%$ & $15 \%$ & $13 \%$ \\
\hline Complaint at dominant arm & $50 \%$ & $44 \%$ & $47 \%$ \\
\hline \multicolumn{4}{|l|}{ Duration of current episode } \\
\hline$<1$ week & $5 \%$ & $7 \%$ & $7 \%$ \\
\hline 1week-1 month & $36 \%$ & $32 \%$ & $33 \%$ \\
\hline $1-6$ months & $29 \%$ & $37 \%$ & $34 \%$ \\
\hline$>6$ months & $30 \%$ & $24 \%$ & $26 \%$ \\
\hline History of elbow complaints & $51 \%$ & $43 \%$ & $45 \%$ \\
\hline Musculoskeletal co-morbidity & $56 \%$ & $47 \%$ & $50 \%$ \\
\hline Pain intensity [scale $0-10$; mean, (SD)] & $6.0(1.9)$ & $4.4(2.1)^{* *}$ & $4.8(2.2)$ \\
\hline Functional disability [scale $0-100 ;$ median (IR)] & $36.3(28.8)$ & $16.5(21.3)^{* *}$ & $20.0(25.3)$ \\
\hline \multicolumn{4}{|l|}{ Work-related factors } \\
\hline Heavy physical work [scale $0-100 ;$ mean (SD)] & $36.1(40.2)$ & $16.7(36.1)^{* *}$ & $22.2(42.8)$ \\
\hline Static postures and repetitive movements (scale $0-100$ ) & $55.6(47.2)$ & $44.4(44.4) * *$ & $44.4(38.9)$ \\
\hline Sitting for a long period of time & $22 \%$ & $48 \% * *$ & $42 \%$ \\
\hline VDU-work for a long period of time & $21 \%$ & $36 \% *$ & $31 \%$ \\
\hline Decision authority [scale 3-12; mean (SD)] & $9.0(3.0)$ & $9.0(3.0)^{*}$ & $9.0(3.0)$ \\
\hline Job demands [scale 5-20; mean (SD)] & $13.4(3.6)$ & $12.8(2.9)$ & $12.9(3.1)$ \\
\hline Co-worker support [scale 4-16; mean (SD)] & $12.0(1.0)$ & $12.0(2.0)$ & $12.0(2.0)$ \\
\hline Job security & $78 \%$ & $85 \%$ & $83 \%$ \\
\hline Job as perceived cause of complaint & $62 \%$ & $49 \%$ & $56 \%$ \\
\hline
\end{tabular}


Table 3 Relationship between work-related risk factors and sick leave in patients with neck or upper extremity complaints: results from the multiple Cox regression analyses

\begin{tabular}{|c|c|c|c|c|c|}
\hline Determinant & $N$ & Crude HR & $95 \% \mathrm{CI}$ & Adjusted HR & $95 \% \mathrm{CI}$ \\
\hline Heavy physical work (per $10 \%$ increase) & $69 / 320$ & 1.23 & {$[1.12 ; 1.36]^{*}$} & & \\
\hline For patients with low scores on worrying ${ }^{d}$ & $21 / 140$ & & & 1.05 & {$[0.93 ; 1.18]^{\mathrm{a}}$} \\
\hline For patients with high scores on worrying ${ }^{\mathrm{d}}$ & $48 / 180$ & & & 1.32 & {$[1.07 ; 1.62]^{\mathrm{a}, *}$} \\
\hline Static postures, repetitive movements (per $10 \%$ increase) & $69 / 320$ & 1.16 & {$[1.06 ; 1.27]^{*}$} & 1.04 & {$[0.94 ; 1.14]^{\mathrm{b}}$} \\
\hline Sitting for long periods of time (vs. never/now and then) & $16 / 322$ & 0.40 & {$[0.23 ; 0.70]^{*}$} & & \\
\hline For patients with low scores on worrying ${ }^{\mathrm{d}}$ & $2 / 180$ & & & 0.81 & {$[0.43 ; 1.52]^{\mathrm{a}}$} \\
\hline For patients with high scores on worrying ${ }^{\mathrm{d}}$ & $14 / 142$ & & & 0.17 & {$[0.04 ; 0.72]^{\mathrm{a}, *}$} \\
\hline VDU-work long periods of time (vs. never/now and then) & $15 / 322$ & 0.56 & {$[0.32 ; 1.00]^{*}$} & 0.70 & {$[0.36 ; 1.36]^{\mathrm{c}}$} \\
\hline \multicolumn{6}{|l|}{ Decision authority } \\
\hline Low & & 1.00 & & & \\
\hline Medium & & 0.80 & {$[0.47 ; 1.35]$} & 0.94 & {$[0.55 ; 1.59]^{\mathrm{a}}$} \\
\hline High & & 0.59 & {$[0.31 ; 1.13]$} & 0.87 & {$[0.45 ; 1.68]^{\mathrm{a}}$} \\
\hline \multicolumn{6}{|l|}{ Job demands } \\
\hline Low & $23 / 115$ & 1.00 & & 1.00 & \\
\hline Medium & $20 / 111$ & 0.90 & {$[0.49 ; 1.64]$} & 0.91 & {$[0.50 ; 1.66]^{\mathrm{a}}$} \\
\hline High & $27 / 95$ & 1.42 & {$[0.81 ; 2.48]$} & 1.13 & {$[0.64 ; 1.97]^{\mathrm{a}}$} \\
\hline \multicolumn{6}{|l|}{ Co-worker support } \\
\hline Low & $16 / 86$ & 1.00 & & 1.00 & \\
\hline Medium & $31 / 140$ & 1.19 & {$[0.65 ; 2.18]$} & 1.22 & {$[0.67 ; 2.23]^{\mathrm{a}}$} \\
\hline High & $23 / 88$ & 1.40 & {$[0.74 ; 2.66]$} & 1.14 & {$[0.59 ; 2.17]^{\mathrm{a}}$} \\
\hline Low job security (vs high) & $70 / 319$ & 1.43 & {$[0.78 ; 2.60]$} & 1.47 & {$[0.80 ; 2.69]^{\mathrm{a}}$} \\
\hline \multicolumn{6}{|l|}{ Job strain } \\
\hline High decision authority, low job demands & $10 / 77$ & 1.00 & & 1.00 & \\
\hline High decision authority, high job demands & $11 / 52$ & 1.63 & {$[0.69 ; 3.84]$} & 1.29 & {$[0.54 ; 3.07]^{\mathrm{b}}$} \\
\hline Low decision authority, low job demands & $25 / 104$ & 1.85 & {$[0.89 ; 3.85]$} & 1.30 & {$[0.62 ; 2.73]^{\mathrm{b}}$} \\
\hline Low decision authority, high job demands & $24 / 86$ & 2.15 & {$[1.03 ; 4.49]^{*}$} & 1.15 & {$[0.54 ; 2.47]^{\mathrm{b}}$} \\
\hline
\end{tabular}

$N$ Number of patients reporting sick leave/total, $H R$ hazard ratio

$* P<0.05$

${ }^{a}$ Adjusted for sick leave at baseline and sex

b Adjusted for sick leave at baseline, sex and worrying

${ }^{c}$ Adjusted for sick leave at baseline, sex, education level, quality of life, working in static postures and with repetitive movements

${ }^{\mathrm{d}}$ Effect modification by worrying

work. In patients scoring high on "worrying" the relative risk of sick leave was 1.32 (95\% CI 1.07-1.62) per $10 \%$ increase in heavy physical work. Patients who were sitting for long periods of time had a reduced risk of sick leave as compared to patients who did not spent a lot of time sitting, again only in patients who scored high on "worrying" $(\mathrm{HR}=0.17,95 \% \mathrm{CI} 0.04-0.72)$. In patients who scored low on "worrying", sitting for long periods of time did not have an effect on sick leave $(P=0.43)$. Sick leave at baseline, sex and musculoskeletal co-morbidity did not modify the relationship between the physical and psychosocial risk factors and sick leave. The psychosocial risk factors neither modified the relationship between heavy physical work and sick leave.

\section{Discussion}

We studied a population of working employees who consulted their GP with a complaint at the neck or upper extremity. The results show that heavy physical work and prolonged sitting in patients that worry a lot predicted sick leave in the three successive months after baseline. Other physical and psychosocial risk factors appeared not to be related to sick leave after adjustment for confounding variables.

Individual factors and stress may have a modifying effect on the relation between work related factors and outcome in musculoskeletal complaints. The pain coping style "worrying" appeared to be an effect modifier in the 
relationship between heavy physical work and sick leave. An increase of $10 \%$ of heavy physical work in patients who worried a lot increased the probability on sick leave with 1.3. A passive coping style like worrying (e.g., "focus on pain all the time"; "I think the pain will get worse") is thought to generate a preoccupation with bodily symptoms, which may increase the perception of pain and disability (Covic et al. 2000; Evers et al. 2003) or generate a different appraisal of the work situation and musculoskeletal symptoms (Bongers et al. 2002b), which in turn may lead to sick leave. Sitting for long periods of time reduced the risk of sick leave, only in patients who scored high on the pain subscale "worrying" In the study of Ariëns et al. (2002) sitting was also related to a reduced risk of sick leave. However, uncomfortable sitting has been found to increase the number of days on sick leave due to neck and shoulder disorders (Ekberg and Wildhagen 1996). Sitting was highly negatively correlated with heavy physical work. Perhaps, people who sit for long periods of time at their work do not experience heavy physical work, and thus the absence of heavy physical work results in a reduced risk. This may explain why sitting had only a protective effect in patients that worry a lot.

In the unadjusted analyses, all the physical risk factors and two of the psychosocial risk factors (i.e., decision authority and job strain) had a statistically significant effect on sick leave. It is partly due to the underlying distribution of the exposure which physical factors are most strongly associated with sick leave in the multivariate analyses. Adjustment for sick leave at baseline had the strongest effect on the presented associations, diminishing the strength of the relations between work-related risk factors and sick leave. Sick leave may have been the result of exposure to these risk factors, but once patients were on sick leave work-related risk factors no longer seem to have an impact on continued sick leave. Half of the patients who reported sick leave within the 3 months before baseline also reported sick leave in the 3 months following baseline. The effect of sick leave in the months preceding baseline apparently outweighed the impact of work-related risk factors in the analyses. The proportion of patients reporting sickness during the 3 months follow-up was too small to be able to exclude patients reporting sick leave at baseline. Cox regression produces large estimates of the standard errors, which results in conservative estimates of the confidence intervals (Skov et al. 1998). Therefore, it may be possible that relevant associations did not reach statistical significance.

Sick leave data were collected by a self-reported questionnaire. Sick leave data collected by an objective method (i.e., sick leave records) may be more accurate than selfreported sick leave. However, in large epidemiological studies the use of questionnaires is more feasible and can be a source of reliable data on sick leave. Burdorf et al. (1996) investigated the reliability of self-reported sick leave with company records as reference. A good agreement was found between prevalence, frequency and duration of self reported sick leave and company records in patients with back pain. In our study we only examined the prevalence of sick leave and consider the reported sick leave data to be fairly reliable.

Our study population differs from an occupational cohort, consisting of workers from a wide variety of occupational settings who had visited the GP due to neck or upper extremity complaints. This makes our results more widely generalisable than a selective sample of workers from a specific company or industry. However, due to the fact that our population already had symptoms at baseline we may not be able to discriminate between factors that are a consequence of sick leave at baseline and factors that may increase the risk of sick leave. For example, patients may worry more about their pain problem because they are no longer able to work, or worrying about their pain problem may be the reason for sick leave after their visit to the doctor.

In the Netherlands the GP is often confronted with patients who are on sick leave due to musculoskeletal complaints, as patients who seek medical care usually first consult their GP. The GP acts as a gatekeeper in the health care system. At the time of our study, referrals to the second or third level of care could, in principle, only be made by the GP. This is comparable to, for instance, the British and the Canadian health care systems. As a result of their position in the Dutch health care system, GPs could play an important role in the prevention of aggravation of complaints. In this study the copying style "worrying" seemed to be an important factor in patients who have jobs involving heavy physical work. Reassuring patients might help to reduce aggravation of complaints, which might improve the chances of return to work in this group of patients with neck or upper extremity complaints. It may be interesting to investigate whether early intervention aimed at promoting particular coping styles can prevent or reduce sick leave in patients with neck or upper extremity complaints in a primary care setting.

In conclusion, heavy physical work increased the risk of sick leave and prolonged sitting reduced the risk of sick leave in a subgroup of patients who worry much. Other work-related physical risk factors and workrelated psychosocial risk factors were not significantly related to sick leave. Additional large longitudinal studies of sufficiently large size among employees with neck or upper extremity complaints are needed to confirm our results. 
Acknowledgments This study is financially supported by the Dutch Arthritis Association.

\section{References}

American College of Sports Medicine Position Stand (1990) American College of Sports Medicine Position Stand. The recommended quantity and quality of exercise for the developing and maintaining cardiorespiratory and muscular fitness in healthy adults. Med Sci Sports Exerc 22:265-274

Ariëns GA, van Mechelen W, Bongers PM, Bouter LM, van der Wal G (2000) Physical risk factors for neck pain. Scand J Work Environ Health 26:7-19

Ariëns GA, van Mechelen W, Bongers PM, Bouter LM, van der Wal G (2001) Psychosocial risk factors for neck pain: a systematic review. Am J Ind Med 39:180-193

Ariëns GA, Bongers PM, Hoogendoorn WE, van der Wal G, van Mechelen W (2002) High physical and psychosocial load at work and sickness absence due to neck pain. Scand J Work Environ Health 28:222-231

Barros AJ, Hirakata VN (2003) Alternatives for logistic regression in cross-sectional studies: an empirical comparison of models that directly estimate the prevalence ratio. BMC Med Res Methodol $3: 21$

Bernard BP (1997) Musculoskeletal disorders and workplace factors: a critical review of epidemiologic evidence for work-related musculoskeletal disorders of the neck, upper extremity, and low back. Publication no. 97-141. National Institute for Occupational Safety and Health, Cincinnati

Blatter BM, Bongers P (1999) Work related neck and upper limb symptoms (RSI): high risk occupations and risk factors in the Dutch working population. TNO-report 4070117/r9800293. TNO Work and Employment, Hoofddorp

Blatter BM, van den Heuvel SG, Bongers PM, Picavet HSJ, Schoemaker CG (2001) De omvang van verzuim en arbeidsongeschiktheid door RSI [The prevalence of work leave and work disability due to RSI]. Doetinchem: Arboconvenant, Ministerie van Sociale Zaken en Werkgelegenheid, 's-Gravenhage/Elsevier

Bongers PM, de Vet HC, Blatter BM (2002a) [Repetitive strain injury (RSI): occurrence, etiology, therapy and prevention]. Ned Tijdschr Geneeskd 146:1971-1976

Bongers PM, Kremer AM, ter Laak J (2002b) Are psychosocial factors, risk factors for symptoms and signs of the shoulder, elbow, or hand/wrist? A review of the epidemiological literature. Am J Ind Med 41:315-342

Borghouts JA, Koes BW, Vondeling H, Bouter LM (1999) Cost-ofillness of neck pain in The Netherlands in 1996. Pain 80:629636

Bot SDM, Terwee CB, van der Windt DAWM, Feleus A, BiermaZeinstra SM, Knol DL, Bouter LM, Dekker J (2004) Internal consistency and validity of a new physical workload questionnaire. Occup Environ Med 61:980-986

Burdorf A, Post W, Bruggeling T (1996) Reliability of a questionnaire on sickness absence with specific attention to absence due to back pain and respiratory complaints. Occup Environ Med 53:58-62

Cole DC, Hudak PL (1996) Prognosis of nonspecific work-related musculoskeletal disorders of the neck and upper extremity. Am J Ind Med 29:657-668

Covic T, Adamson B, Hough M (2000) The impact of passive coping on rheumatoid arthritis pain. Rheumatology (Oxford) 39:10271030

Ekberg K, Wildhagen I (1996) Long-term sickness absence due to musculoskeletal disorders: the necessary intervention of work conditions. Scand J Rehabil Med 28:39-47
Eriksen W, Natvig B, Knardahl S, Bruusgaard D (1999) Job characteristics as predictors of neck pain. A 4-year prospective study. J Occup Environ Med 41:893-902

Evers AW, Kraaimaat FW, Geenen R, Jacobs JW, Bijlsma JW (2003) Pain coping and social support as predictors of long-term functional disability and pain in early rheumatoid arthritis. Behav Res Ther 41:1295-310

Feuerstein M, Huang GD, Haufler AJ, Miller JK (2000) Development of a screen for predicting clinical outcomes in patients with work-related upper extremity disorders. J Occup Environ Med 42:749-61

Hagg GM, Astrom A (1997) Load pattern and pressure pain threshold in the upper trapezius muscle and psychosocial factors in medical secretaries with and without shoulder/neck disorders. Int Arch Occup Environ Health 69:423-32

Hansson T, Jensen I (2004) Swedish Council on Technology Assessment in Health Care (SBU), Chapter 6. Sickness absence due to back and neck disorders. Scand J Public Health Suppl 63:109-51

IJzelenberg W, Molenaar D, Burdorf A (2004) Different risk factors for musculoskeletal complaints and musculoskeletal sickness absence. Scand J Work Environ Health 30:56-63

Jones JR, Hodgson JT, Clegg TA, Elliott RC (1998) Self-reported work-related illness in 1995. Results from a household survey. HMSO, London, pp 180

Karasek RA (1979) job demands, job decision latitude, and mental strain: implications for job redesign. Adm Sci Q 24:285-307

Karasek R, Brisson C, Kawakami N, Houtman I, Bongers P, Amick B (1998) The job content questionnaire (JCQ): an instrument for internationally comparative assessments of psychosocial job characteristics. J Occup Health Psychol 3:322-55

Kraaimaat FW, Evers AW (2003) Pain-coping strategies in chronic pain patients: psychometric characteristics of the pain-coping inventory (PCI). Int J Behav Med 10:343-363

Kraaimaat F, Bakker A, Evers A (1997) Pain coping strategies in chronic pain patients: the development of the Pain Coping Inventory (PCI). Gedragstherapie 30:185-201

Lee J, Chia KS (1994) Use of the prevalence ratio $v$ the prevalence odds ratio as a measure of risk in cross sectional studies. Occup Environ Med 51:841

Lundberg U (2002) Psychophysiology of work: stress, gender, endocrine response, and work-related upper extremity disorders. Am J Ind Med 41:383-392

Malchaire J, Cock N, Vergracht S (2001) Review of the factors associated with musculoskeletal problems in epidemiological studies. Int Arch Occup Environ Health 74:79-90

Muggleton JM, Allen R, Chappell PH (1999) Hand and arm injuries associated with repetitive manual work in industry: a review of disorders, risk factors and preventive measures. Ergonomics 42:714-739

Rissen D, Melin B, Sandsjo L, Dohns I, Lundberg U (2000) Surface EMG and psychophysiological stress reactions in women during repetitive work. Eur J Appl Physiol 83:215-222

Schellevis FG, Westert GP, de Bakker DH, Groenewegen PP, van der Zee J, Bensing JM (2003) De tweede nationale studie naar ziekten en verrichtingen in de huisartsenpraktijk: aanleiding en methoden [The second Dutch national survey of general practice: cause and methods]. Huisarts en Wetenschap 46(1):7-12

Skov T, Deddens J, Petersen MR, Endahl L (1998) Prevalence proportion ratios: estimation and hypothesis testing. Int $\mathrm{J}$ Epidemiol 27:91-95

Terluin B (1998) The four dimensional symptom questionnaire (4DSQ) in general practice. De Psycholoog 33:18-24

Thompson ML, Myers JE, Kriebel D (1998) Prevalence odds ratio or prevalence ratio in the analysis of cross sectional data: what is to be done? Occup Environ Med 55:272-277

van der Waal JM, Bot SD, Terwee CB, van der Windt DA, Bouter LM, Dekker J (2003) Determinants of the clinical course of 
musculoskeletal complaints in general practice: design of a cohort study. BMC Musculoskelet Disord 4:3

van der Windt DA, Thomas E, Pope DP, de Winter AF, Macfarlane GJ, Bouter LM, Silman AJ (2000) Occupational risk factors for shoulder pain: a systematic review. Occup Environ Med $57: 433-442$
Ware JE Jr, Sherbourne CD (1992) The MOS 36-item short-form health survey (SF-36). I. Conceptual framework and item selection. Med Care 30:473-483

Zakaria D, Robertson J, MacDermid J, Hartford K, Koval J (2002) Work-related cumulative trauma disorders of the upper extremity: navigating the epidemiologic literature. Am J Ind Med 42:258-269 